\title{
Transient intensity changes of cosmic rays beyond the heliospheric termination shock as observed at Voyager 1
}

\author{
W. R. Webber, ${ }^{1}$ A. C. Cummings, ${ }^{2}$ F. B. McDonald, ${ }^{3}$ E. C. Stone, ${ }^{2}$ B. Heikkila, ${ }^{4}$ \\ and N. Lal ${ }^{4}$ \\ Received 9 February 2009; revised 30 March 2009; accepted 28 May 2009; published 30 July 2009.
}

[1] This paper continues our studies of temporal variations of cosmic rays beyond the heliospheric termination shock (HTS) using Voyager 1 (V1) data when V1 was beyond 94 AU. This new study utilizes cosmic ray protons and electrons of several energies. Notable transient decreases of $5-50 \%$ are observed in galactic cosmic ray nuclei and electrons at V1 shortly after similar decreases are observed at Voyager 2 (V2) still inside the HTS. These decreases at V1 appear to be related to the large solar events in September 2005 and December 2006 and the resulting outward moving interplanetary shock. These two large interplanetary shocks were the largest observed at V2 after V1 crossed the HTS at the end of 2004. They were observed at V2 just inside the HTS at 2006.16 and 2007.43 providing timing markers for V1. From the timing of the intensity decreases observed at V1 as the shocks first reach the HTS and then later reach V1 itself, we can estimate the shock speed beyond the HTS to be between 240 and $300 \mathrm{~km} \mathrm{~s}^{-1}$ in both cases. From the timing of the decreases observed when the shock first reaches the HTS and then several months later encounters the heliopause, we can estimate the heliosheath thickness to be $31 \pm 4$ and $37 \pm 6 \mathrm{AU}$, respectively, for the two sequences of three decreases seen at V1. These values, along with the distances to the HTS that are determined, give distances from the Sun to the heliopause of $121 \pm 4$ and $124 \pm 6$ AU, respectively.

Citation: Webber, W. R., A. C. Cummings, F. B. McDonald, E. C. Stone, B. Heikkila, and N. Lal (2009), Transient intensity changes of cosmic rays beyond the heliospheric termination shock as observed at Voyager 1, J. Geophys. Res., 114, A07108, doi:10.1029/2009JA014156.

\section{Introduction}

[2] Short-term (transient) time variations of cosmic rays of a few days to weeks play an important role in understanding the overall modulation of cosmic ray intensity throughout the heliosphere by the outflowing solar wind plasma and magnetic fields. Transient decreases at the Earth, also know as Forbush decreases, are an important part of the overall 11-year solar modulation cycle for galactic cosmic rays (GCR) [Lookwood, 1960]. As the Voyager 1 and 2 (V1 and V2) spacecraft have moved outward in the heliosphere from 1977 to 2004, they have observed the largest of these transients moving outward at average speeds of $\sim 500-800 \mathrm{~km} \mathrm{~s}^{-1}$. Individual events at the Earth may coalesce into merged interaction regions (MIR) and even global merged interaction regions (GMIR)

\footnotetext{
${ }^{1}$ Department of Astronomy, New Mexico State University, Las Cruces, New Mexico, USA.

${ }^{2}$ Downs Laboratory, California Institute of Technology, Pasadena, California, USA.

${ }^{3}$ Institute of Physical Science and Technology, University of Maryland, College Park, Maryland, USA.

${ }^{4}$ NASA Goddard Space Flight Center, Greenbelt, Maryland, USA.

Copyright 2009 by the American Geophysical Union. 0148-0227/09/2009JA014156\$09.00
}

with the result that the overall cosmic ray intensity decrease from these shock-related events at V1 and V2, some 30$80 \mathrm{AU}$ from the Sun, is comparable to that observed at the Earth and contributes significantly to the observed longerterm modulation [e.g., Burlaga et al., 1991]. A major example of such a propagating transient decrease is the large event seen at the Earth in late October 2003 (the Halloween event) and about 6-8 months later at V2 and V1 at distances of 73 and $92 \mathrm{AU}$, respectively [Richardson et al., 2005; Intriligator et al., 2005]. Shortly after this event reached V1, near the end of 2004, V1 crossed the heliospheric termination shock (HTS) at a distance of $\sim 94$ AU. Another very large transient intensity decrease was observed later at V2 at 2006.2 [Richardson et al., 2006], most likely related to intense solar activity in August September 2005, about 6 months earlier. This event was also observed at V1 later in 2006 when it was beyond the HTS and marked the beginning of the study of these transient events beyond the HTS in the heliosheath [Webber et al., 2007].

[3] Near the HTS and beyond there are several populations of energetic particles [e.g., Cummings et al., 2006]. They include (1) galactic cosmic rays (GCR) (e.g., H, He) at energies above $\sim 100 \mathrm{MeV}$ nucleon $^{-1}$, (2) anomalous cosmic rays (ACR) (e.g., $\mathrm{H}, \mathrm{He}, \mathrm{O}$ ) in the energy range 


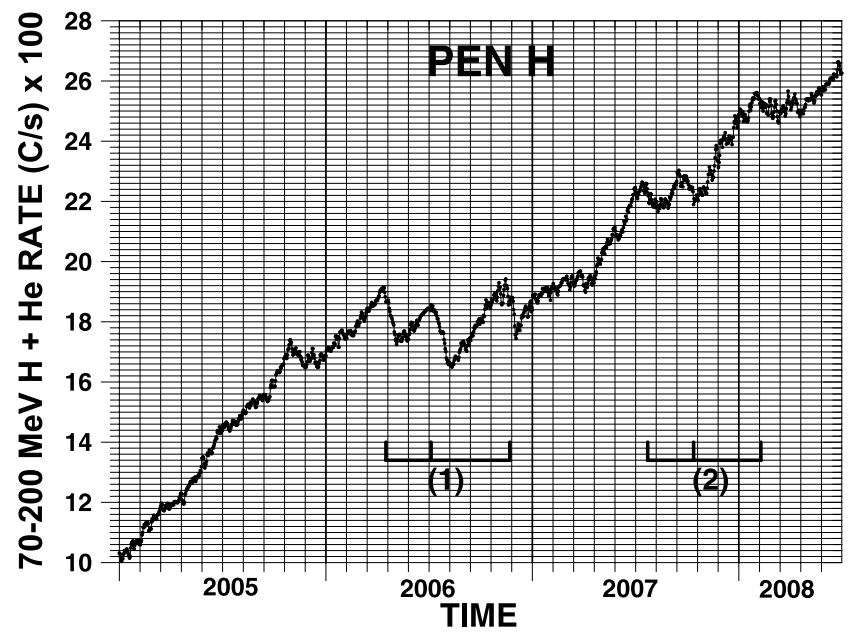

Figure 1. Five-day running average rates from 2005.0 to 2008.5 for the Pen H channel on V1, which is sensitive to both ACR and GCR in the energy range from $\sim 70$ to $200 \mathrm{MeV}$.

from $\sim 10-20 \mathrm{MeV}$ nucleon $^{-1}$ up to $\sim 100 \mathrm{MeV}$ nucleon $^{-1}$, and (3) electrons of energy 6-14 MeV and above. Each of these populations may have a different source region and so might be expected to react differently as these transient events propagate through the heliosheath. The large transient decrease observed at V2 at 2006.2 noted above, and later followed through the heliosheath by studying its effects at V1 [Webber et al., 2007], supports the idea that the heliosheath is not a simple uniformly turbulent region but contains, superimposed, notable large features and structure.

[4] It is the purpose of this paper to examine these temporal intensity features at V1 from the time V1 crossed the HTS at 94 AU at the end of 2004 up to 2008 when V1 was at $\sim 105 \mathrm{AU}$ and about $20 \mathrm{AU}$ beyond the estimated HTS location of $85 \mathrm{AU}$ at that time [Webber, 2005]. A total of nine specific increases/decreases are considered that meet our criteria. Data from V2 between $\sim 75$ and 85 AU during this time period are used as a reference for conditions inside the HTS. After 2007.66, when V2 also encountered the HTS [Stone et al., 2008], both spacecraft are in the heliosheath, one at a heliolatitude $+35^{\circ}(\mathrm{V} 1)$ and the other at $-27^{\circ}(\mathrm{V} 2)$.

\section{V1 and V2 Data and Shock Propagation Inside and Outside the HTS}

[5] Before presenting specific data we should note that most of the particle intensities were basically near their minimum just before V1 crossed the HTS at the end of 2004 [McDonald et al., 2006]. Thus, the intensity increase observed in all plots after 2005.0 represents, in part at least, 11-year solar cycle modulation effects. At the Earth the 11-year solar cycle intensity increase after 2004 was observed to initially reach a broad intensity maximum for GCR and ACR at about 2008.0 according to data from the ACE spacecraft (http://www.srl.caltech.edu/ACE/). This maximum has continued up to the present time near the end of 2008. At V1 this intensity maximum would be expected to be delayed by $\sim 1$ year or more because of propagation effects of the solar wind and would therefore not be expected to arrive at V1 until late 2009 at the earliest.
[6] Three-dimensional simulations of interplanetary (IP) shock propagation in the heliosphere inside the HTS show clearly that the interplanetary shocks generally propagate to the HTS with a symmetric like speed to the locations of V1 at $+35^{\circ}$ and $\mathrm{V} 2$ at $-27^{\circ}$. The detailed features at the north and south latitudes may be different, however, in large part because of the continuous nature of the inputs to the model, which emphasize the longitudinal differences in the location of these spacecraft [Intriligator et al., 2008]. As these shocks approach the HTS, the compressed magnetic field lines, now more or less parallel to the shock surface, will modify energetic particle transmission across the HTS. This variable transmission is one source for the high variability of termination shock particles observed by the V1 spacecraft when it was near but inside the HTS, such as in the $2002-$ 2004 time periods [e.g., Giacalone and Jokipii, 2006].

[7] The V1 spacecraft, when it is in the heliosheath between the HTS and heliopause (HP), is in a previously unexplored region in which the energetic particle entry may be strongly governed by particle transmission across the localized HTS and HP boundary regions that are most directly connected to V1. As the IP shocks encounter the HTS, for example, particle transmission across the HTS in these regions may be temporarily modified, leading directly to intensity changes observed nearly simultaneously at V1 [e.g., Giacalone and Jokipii, 2006]. Similar types of intensity changes are observed at the Earth when shock structures pass near the Earth.

[8] In the absence of current models describing these effects at V1, which are being presented for the first time here, the actual temporal variations that are observed may be utilized to understand the global physics involved. The lack of corresponding V1 magnetic field and plasma data obviously limits this interpretation as well.

[9] With this discussion as a background we show in Figure 1 the intensity-time profile for 70 - to $200-\mathrm{MeV} \mathrm{H}$ nuclei at V1 from 2005.0 through 2008. This channel for V1 contains both anomalous cosmic rays (ACR) and galactic cosmic rays, increasing from a low intensity at the time of the shock crossing. Most of the transient changes we are interested in at V1 show up well in this $\mathrm{H}$ nuclei channel and are marked in Figure 1. The most prominent of these transient changes are (1) a sequence of three intensity decreases starting at $2006.29,2006.51$, and 2006.89, which may be related (as discussed by Webber et al. [2007]); and (2) another sequence of three weaker-intensity decreases starting at 2007.56, 2007.78, and 2008.10, which may also be related. These times are uncertain to \pm 0.02 year and may vary slightly from channel to channel.

[10] The intensity-time record for $\operatorname{GCR}(>70 \mathrm{MeV})$ at V1 is shown in Figure 2. This channel contains a background of a few percent ACR. Here all of the decreases noted above are also observed but with differing magnitudes.

[11] In Figure 3 we show the intensity time profiles for 6- to $14-\mathrm{MeV}$ electrons, believed to be GCR. Here again all of the decreases seen for ACR and GCR are strongly observed by electrons as well.

\section{Discussion of Individual Events}

[12] In order to be considered significant, intensity decreases/increases must be seen at V1 simultaneously (to 


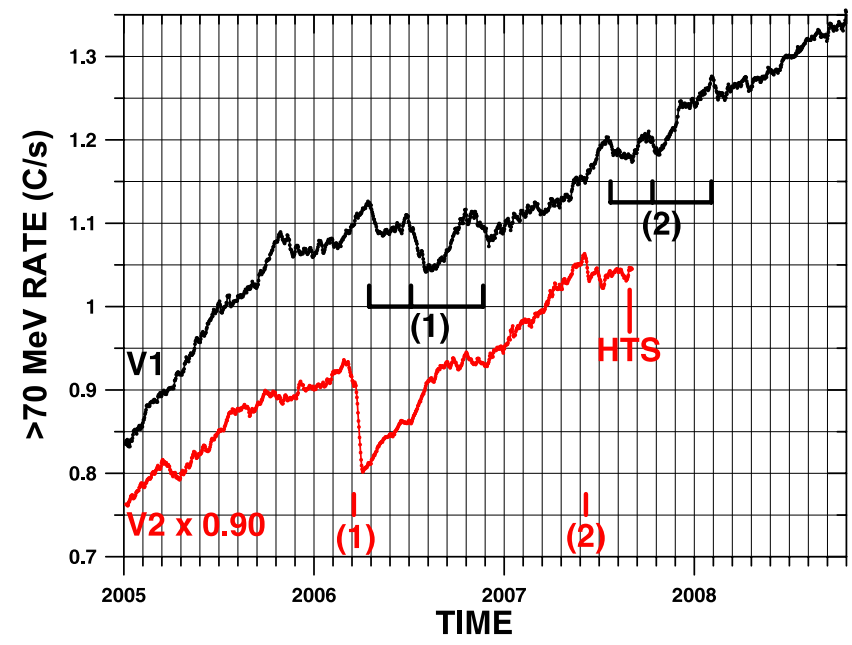

Figure 2. Five-day running average rate of the Pen L channel $(>70 \mathrm{MeV})$ at V1 and V2 from 2005.0 to 2008.8 . This rate is mostly GCR with a maximum $5-10 \%$ background of ACR $\mathrm{H}>70 \mathrm{MeV}$.

\pm 0.02 year) in all three channels (GCR, ACR, and electrons) and must be larger than $2-3 \%$. In the time period from the V1 HTS crossing at $\sim 2005.0$ to mid-2008, a total of nine events met these selection criteria. The largest of these intensity changes, two groups of three events, comprise the two sequences described here. The other events are weaker or have different intensity-time profiles and are not believed to be part of these two sequences. They are described separately in Appendix A. At V2 over the same time period, only two intensity changes meet these same criteria, the two intensity decreases that we argue are the triggers for the two sequence events at V1.

[13] The first sequence (sequence 1) of three decreases seen in all channels at 2006.29, 2006.57, and 2006.89 at V1 has already been discussed in an earlier paper [Webber et al., 2007]. Here we provide some additional details. Webber et al. associated these decreases at V1 with a giant transient GCR decrease seen at V2 at 79 AU starting at 2006.19 and an interplanetary shock observed to start at 2006.16 [Richardson et al., 2006; Webber et al., 2007]. The interplanetary shock and/or GMIR observed at V2 at this time is the largest seen in this solar cycle in the outer heliosphere. The first cosmic ray decrease at V1 related to this event was observed at $2006.29 \pm 0.02$, just 0.10 year after the decrease at V2. The travel time of 0.10 year between the decrease at V2 and this initial decrease at V1, (assuming an HTS distance of $89 \mathrm{AU}$ at $+35^{\circ}$ latitude) would be consistent with a N-S spherically symmetric IP shock, traveling at an average speed of $400 \mathrm{~km} \mathrm{~s}^{-1}$, reaching the HTS at $89 \mathrm{AU}$ at 2006.29, which is when the decrease occurred. If instead this initial decrease at $\mathrm{V} 1$ occurs when this shock actually reaches $\mathrm{V} 1$, which is now at a distance $\sim 99 \mathrm{AU}$, this would imply an average speed between V2 and V1 of over $800 \mathrm{~km} \mathrm{~s}^{-1}$, which is even larger than the average speed of this shock of $730 \mathrm{~km} \mathrm{~s}^{-1}$ between the Earth and V2, a most unlikely possibility. This particular timing of the two decreases at V1 and the lack of any other precursor events in the V2 data for either the first or second decrease at V1 lead us to the conclusion that the first two decreases at V1 are due to the IP shock first reaching the HTS at 2006.29 and then later at 2006.51 reaching V1 itself.

[14] For the second decrease in this sequence at $\mathrm{V} 1$ which started at $\sim 2006.51 \pm 0.02$, the travel time between the arrival of this shock at the HTS at 2006.29 and this second decrease has been used to obtain an estimate of the shock speed between the HTS and V1 of $\sim 240 \pm 40 \mathrm{~km} \mathrm{~s}^{-1}$ [Webber et al., 2007].

[15] The lack of any precursor events at V2 in the $>70 \mathrm{MeV}$ GCR, ACR, and 6- to $14-\mathrm{MeV}$ electron channels also leads us to the conclusion that the next decrease at V1 at 2006.89 in all three channels is related to this same initial event. So we have the scenario in which all three decreases at V1 are related to the large transient decrease seen at V2 at about 2006.19. In this scenario the first decrease at V1 is when the IP shock reaches the HTS, the second is when it reaches V1, and the third is when it reaches the HP. Our further conclusions are based on this assumption.

[16] The third decrease in this sequence at V1 started at $2006.89 \pm 0.02$. In both the ACR and GCR channels this decrease was followed by a resumption of the longer-term intensity increase. We believe that this increasing intensity after 2006.89 is important because it marks the passage of the shock beyond the outer modulation boundary, the heliopause (HP), with a consequent weakening of the overall modulation and an increase in intensity.

[17] Associating this third decrease with the arrival of the modified IP shock at the HP, the time delay of 0.38 year between the arrival of this shock at V1 and at the HP, along with an estimated speed of $240 \mathrm{~km} \mathrm{~s}^{-1}$, places the HP distance at about 20 AU beyond $\mathrm{V} 1$, or $120 \pm 3$ AU from the Sun, neglecting possible time delays in the returning signal from the HP or a slowing down of the shock beyond V1 [Webber et al., 2007; Washimi et al., 2007].

[18] The time history of this first sequence starting with the instigating event at V2 is shown schematically in Figure 4.

[19] The next series (sequence 2) of three decreases (seen in all three channels) occurs at $2007.56 \pm 0.02,2007.78 \pm$ 0.02 , and $2008.11 \pm 0.03$, respectively (see also Figure 5).

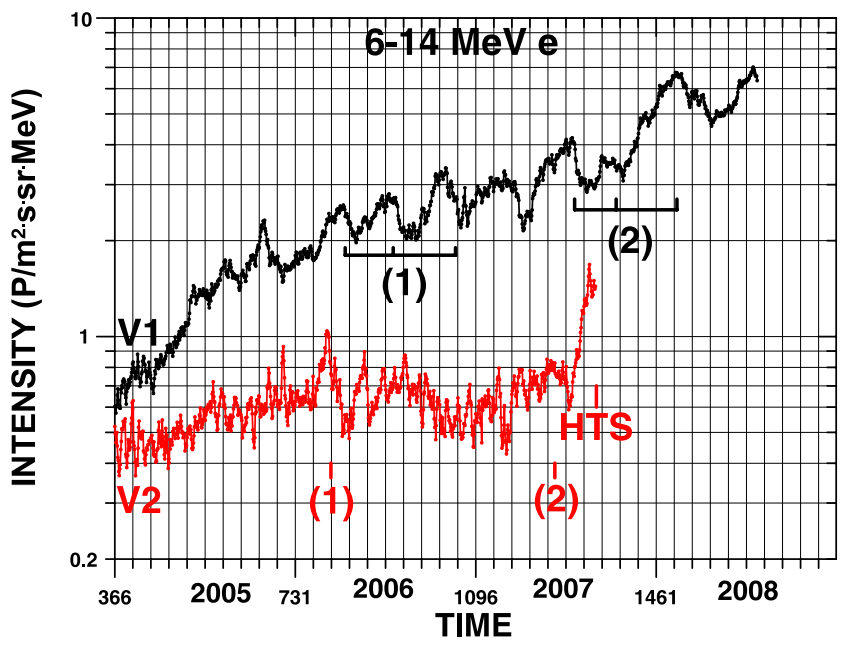

Figure 3. Five-day running average of 6- to $14-\mathrm{MeV}$ electron intensity (GCR) at V1 and V2 from 2005.0 to 2008.8. 


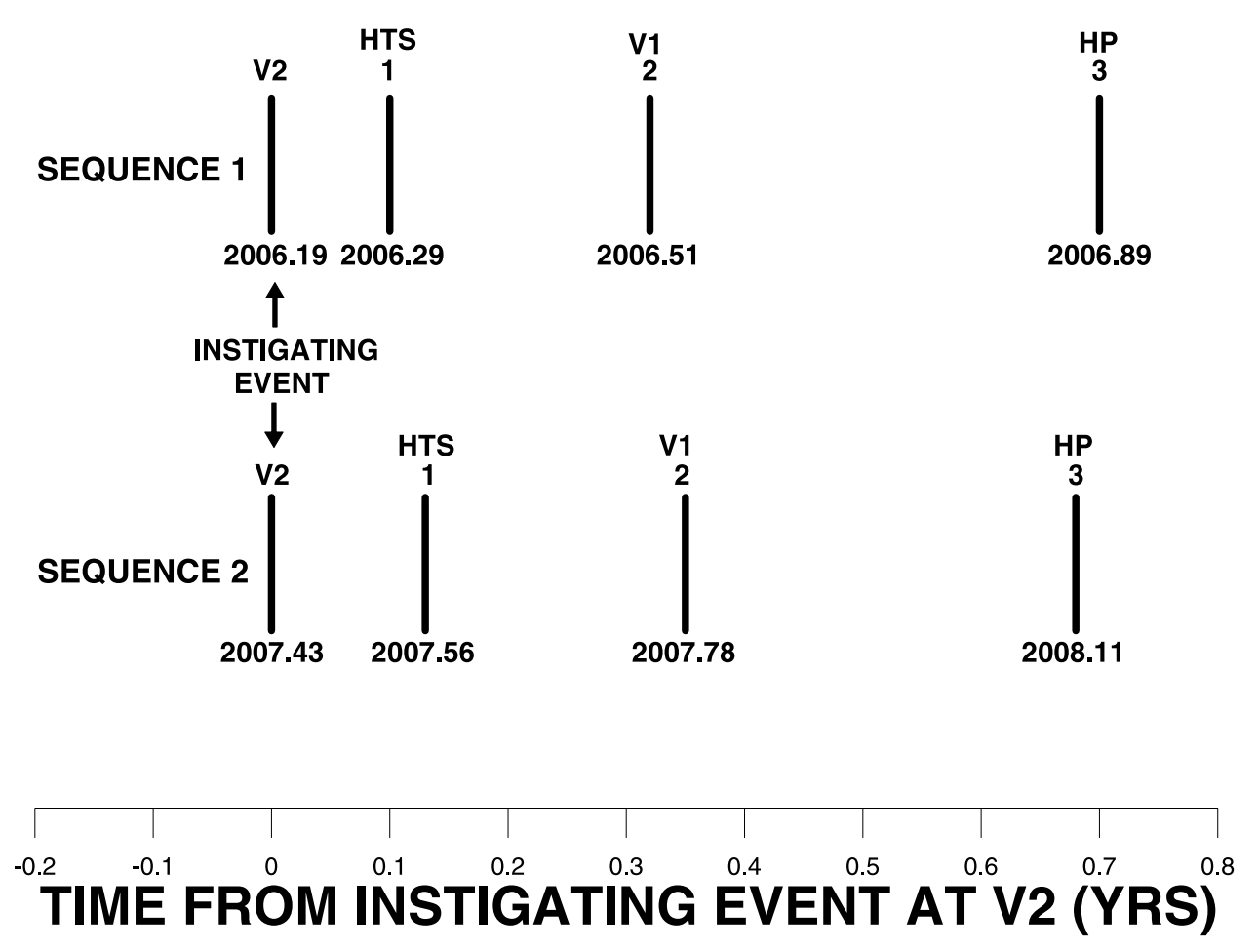

Figure 4. Schematic time history of sequence events 1 and 2 starting with the instigating events for these sequences at V2. Note the striking similarity of these two time sequences at V1.

At 2007.56 V1 was at 103.8 AU, $\sim 13.3$ AU beyond the estimated HTS location of $\sim 90.5 \mathrm{AU}$ at $+35^{\circ}$.

[20] The V2 data associated with this first decrease seen at V1 are very interesting. Significant sudden intensity decreases of $\sim 3 \%$ were seen at 2007.43 and 2007.49 in the $>70 \mathrm{MeV}$ (GCR) channel at V2 (see Figure 5). These intensity decreases at $\mathrm{V} 2$, then at $\sim 83 \mathrm{AU}$, are most likely associated with the December 2006 events at the Earth, $\sim 6$ months earlier. The intensity decrease at the Earth, as observed by neutron monitors, also occurred in two large steps, the first on 7 December of amplitude $\sim 5 \%$ and the second on 13 December of amplitude $\sim 7 \%$. Soon after this event was observed at V2, this spacecraft passed the HTS at 2007.66 at a distance of $283.7 \mathrm{AU}$ and was no longer useful as a timing marker.

[21] The time between the initial decrease at 2007.43 at V2 and the first decrease at $\mathrm{V} 1$ at 2007.56 is $\sim 0.13 \pm 0.02$ year. If this time delay is the time for the shock to travel from V2 to the location of V1 itself at 103.8 AU, this would imply an average speed between V2 and V1 of $\sim 730 \mathrm{~km} \mathrm{~s}^{-1}$, which is comparable to the average speed of $\sim 720 \mathrm{~km} \mathrm{~s}^{-1}$ for the December 2006 shock to travel from the Earth to V2, again a very unlikely possibility. However, if the first decrease at $\mathrm{V} 1$ is due to the arrival of this shock at the HTS (estimated to be at $\sim 90.5 \mathrm{AU}$ at $+35^{\circ}$ at this time [Webber, 2005]), then the average speed between V2 and the HTS is $250-300 \mathrm{~km} \mathrm{~s}^{-1}$ (assuming N-S symmetry), a speed much more compatible with the solar wind speeds actually being measured at V2 at that time [Richardson et al., 2008]. As with sequence 1, because of this specific timing and also a lack of individual precursor events at V2 for the two decreases seen at V1, we are led again to the conclusion that the first decrease at V1 occurs when this shock reaches the HTS at 2007.56, and the second decrease at V1 at 2007.78 occurs when this shock reaches $\mathrm{V} 1$ itself.

[22] The second decrease in this second sequence started at $\sim 2007.78 \pm 0.02$ at $\mathrm{V} 1$. At this time V1 was at $\sim 104.4 \mathrm{AU}$, or $\sim 13$ AU beyond the HTS. The time delay between decreases 1 and 2 of this sequence at V1 is $0.22 \pm 0.02$ year, during which time the shock is presumed to have traveled from the HTS at a distance of 91.0 AU to V1, now at 104.4 AU, for a total distance of $13.4 \pm 0.5 \mathrm{AU}$. The average speed between the HTS and V1 is, thus, determined to be $290 \pm 50 \mathrm{~km} \mathrm{~s}^{-1}$. This speed is roughly the same as

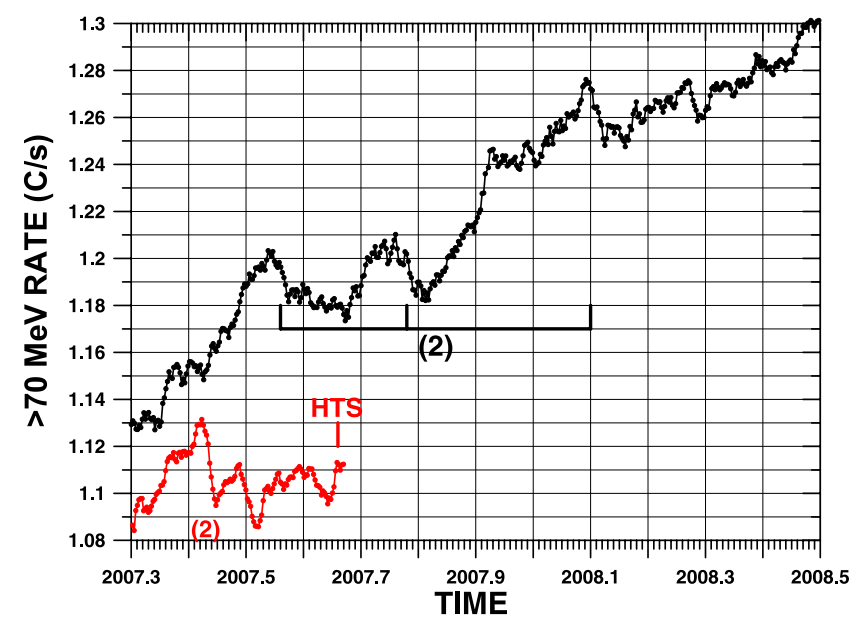

Figure 5. Five-day running average of Pen L rate from 2007.2 to 2008.5 showing higher intensity-time resolution of the sequence event 2 at V2 and V1. 
the average speed of $240 \mathrm{~km} \mathrm{~s}^{-1}$ from the HTS to V1 determined for sequence 1 [Webber et al., 2007] but represents much less of a slowing down of the average shock speed beyond the HTS than for the earlier IP shock.

[23] Again, the lack of any precursor events at V2, which was itself beyond the HTS, in the $>70 \mathrm{MeV}$ GCR and 6- to 14-MeV electron channel leads us to the conclusion that the third decrease, which occurred at V1 at about $2008.11 \pm$ 0.03 , is related to the same initial event. So again, we have the scenario in which all three decreases at $\mathrm{V} 1$ are related to the same initial event at 2007.43 at V2, propagating outward through the heliosheath. This last decrease is weakly seen in GCR and ACR, perhaps because of the underlying rapid increase in intensity. The time delay between the second decrease of this sequence, arriving at $\mathrm{V} 1$ at $104.4 \mathrm{AU}$, and this third decrease is $0.33 \pm 0.03$ year, which, at a speed of $290 \pm 40 \mathrm{~km} \mathrm{~s}^{-1}$, corresponds to a distance of $20 \pm 2 \mathrm{AU}$ beyond $\mathrm{V} 1$, or a total $124 \pm 6 \mathrm{AU}$ for the distance of the HP from the Sun.

[24] The time history of this second sequence event starting with the instigating event at V2 is also shown schematically in Figure 4. Note the striking similarity of the two time histories of the sequences, consistent with the argument that the individual decreases at V1 represent in each case the propagation of a transient disturbance through the heliosheath from the HTS to the HP. It is not possible to duplicate this similarity by a juxtaposition of unrelated events propagating outward past V2 and then V1 in the heliosphere.

[25] It is interesting to also note that the December 2006 instigating event at the Earth for the decreases of sequence 2 at $\mathrm{V} 1$ in the outer heliosphere appears to be the last major transient in solar cycle 23 and is well isolated in time from any other large events at the Sun. The intensities of cosmic rays at the Earth have continued to steadily increase from this time up to the present time in early 2009. After this event passed the HP at 2008.11, a little more than a year after it passed the Earth, the GCR intensities at V1 have increased up to the present day (2009.0).

\section{Overall Discussion and Conclusions}

[26] The sequence events 1 and 2 at V1 are very similar in their timing properties between the three decreases and also in the relative magnitude of the individual decreases in the various channels. From their originating events as they passed V2 inside the HTS, we have identified the first decrease of each of the sequences at V1 as the time the IP shock reaches the HTS at $+35^{\circ}$, the latitude of V1. The second decrease of the two sequences are the times that this shock, now moving more slowly, passes the location of V1. From this timing we have obtained shock speeds beyond the HTS of $240 \pm 40$ and $290 \pm 50 \mathrm{~km} \mathrm{~s}^{-1}$ for sequences 1 and 2, respectively. This shock speed is important not only in the theoretical sense but also for calculating the total distance traveled by an IP shock before it finally reaches the HP.

[27] In the scenario considered here the third decrease of each sequence is the time this shock reaches the vicinity of the HP. After this third decrease in both cases the GCR and ACR intensities at V1 resume a longer-term increase. We believe this is an important indicator that the outward propagating shock reaches the $\mathrm{HP}$ at that time and is no longer as effective as a modulating agent.

[28] In the case of sequence 1 the total time from the first to the third decrease is $0.60 \pm 0.03$ year. For sequence 2 this time interval is $0.55 \pm 0.04$ year. These times are indicated schematically in Figure 4. In this scenario this time is related to the time required for this shock to propagate across the heliosheath from the HTS to the HP. For sequence 1, if we assume a constant speed of $240 \mathrm{~km} \mathrm{~s}^{-1}$, this gives a total heliosheath thickness of $\sim 31 \pm 4$ AU. For sequence 2 the average speed from the HTS to V1 was determined to be $\sim 290 \mathrm{~km} \mathrm{~s}^{-1}$; thus, this time of 0.55 year gives a total heliosheath thickness of $\sim 37 \pm 6 \mathrm{AU}$. The errors in these values do not include possible return time uncertainties between the shock arrival at the HP and its observation at V1 or a possible slowing down of the shock beyond V1.

[29] If one uses the theoretical estimate of the heliosheath thickness to be $0.40 \pm 0.02$ times the distance from the Sun to the HTS as obtained in the study by Müller et al. [2006] and takes a typical HTS distance of 90 AU, then a heliosheath thickness of 34-38 AU is obtained from this theoretical work, which is similar to the above estimates based on timing.

[30] In these scenarios the total time for the shock to travel from the Earth to the HP is 1.15 years, or 420 days, for the shock responsible for sequence 1, assuming the related event at the Earth was on 15 September 2005, 2005.71. For the shock responsible for sequence 2, assuming the related event at the Earth was on 7 December 2006, 2006.935, the total transit time was 1.16 years, or 423 days.

[31] It should be noted that for the two largest $\mathrm{kHz}$ radio events observed by the Iowa group on Voyager, the first in 1983, the second in 1992, the transit times, also assumed to be from the Earth to a HP location, were essentially the same with an average of $415 \pm 5$ days [Gurnett et al., 1993].

[32] In summary, this study of temporal variations beyond the HTS reveals several interesting features. First, for each of the sequence events 1 and 2, three related decreases are observed at V1. Each sequence of three events is directly related to a single, isolated in time, large shock that was observed at V2 about 0.10 to 0.13 year earlier than the first decrease of the sequence seen at V1 (see Figure 4). The first decrease at V1 is believed to be when the shock reaches the HTS, the second is when the (reduced) shock reaches V1, and the third is when this shock reaches the HP. From the time delay between decreases 1 and 2 of each sequence we are able to estimate the average shock speed between the HTS and V1. This turns out to be $\sim 240$ and $290 \mathrm{~km} \mathrm{~s}^{-1}$, respectively, for the two sequences studied; this is less than the average shock speed inside the HTS. Estimates of a total heliosheath thickness of $\sim 31 \pm 4$ and $37 \pm 6$ AU are obtained from the time between decreases 1 and 3 of the two sequences using these average propagation speeds.

[33] The total transit time of the shocks from the Earth to the HP is, thus, determined to be $\sim 420$ and 423 days for sequence events 1 and 2, respectively. These total transit times agree well with the average of 415 days determined earlier by Gurnett et al. [1993] from the total transit times based on the onset of $\mathrm{kHz}$ radio emission when earlier large IP shocks were assumed to reach the HP in 1983 and 1992. These transit times lead to estimated HP distances of 
$120 \pm 4$ and $124 \pm 6 \mathrm{AU}$ for the sequence events 1 and 2, respectively.

\section{Appendix A}

[34] A discussion of three other transient increases/ decreases observed by V1 beyond the HTS but not related to the transients associated with the sequence events is included in this section for completeness.

[35] 1. An increase is seen in all channels at 2005.82. This event precedes the two sets of sequence decreases and has quite different intensity-time characteristics. It is seen most strongly in the lowest-energy ACR channels with an increase by a factor of $\sim 2$ in the $1.9-$ to $2.7-\mathrm{MeV} \mathrm{H}$ nuclei channel. The energy dependence of the increase and the time of maxima of the increase look much like a solar cosmic ray event at the Earth; however, the time scale of the overall increase is $\sim 10$ times longer. At V2 an increase of 6- to $14-\mathrm{MeV}$ electrons and also ACR are consistent with the passage of outward moving shocks between about 2005.60 and 2005.65. Thus, the onset of the increase at $\mathrm{V} 1$ is more consistent with the arrival of these shocks at the HTS, not V1 itself. If this is the correct interpretation, then when this shock structure reaches the HTS, it is the source of freshly accelerated low-energy $\mathrm{H}$ ions, seen at V1 as they propagate outward through the heliosheath.

[36] 2. This is an abrupt decrease in amplitude of 30\% in 6- to $14-\mathrm{MeV}$ electrons at 2007.23 followed by an abrupt recovery at 2007.31. It is also seen in lower-energy ACRs but only weakly, if at all, in GCR. Again this has a different intensity-time behavior, as well as different species dependence than the sequence events. A similar intensity decrease is seen starting at 2007.13 and ending at 2007.20 in 6- to14-MeV electrons at V2. Assuming the decreases at V1 and V2 are related and the decrease at V2 is due to a structure passing that spacecraft, the time delay of only 0.1 year would suggest that the decrease at V1 is due to the arrival of this structure at the HTS, not V1 itself, much like event 1 of Appendix A.

[37] 3. A decrease at $\mathrm{V} 1$ seen at 2008.27, 0.16 year after the last decrease of sequence 2 (when the shock causing this sequence is supposed to have reached the HP). This decrease is seen most strongly in 6- to $14-\mathrm{MeV}$ electrons where its amplitude of $\sim 20 \%$ is comparable to the decrease seen in these electrons at $\sim 2008.13$, when the last decrease of sequence 2 reached the HP. This "additional" decrease is only very weakly seen in GCR and ACR, however.

[38] Acknowledgments. This work is supported at NMSU by subcontract 1257395 from JPL. W.R.W. appreciates discussions with coauthors A.C.C. and F.B.M.

[39] Zuyin Pu thanks the reviewers for their assistance in evaluating this paper.

\section{References}

Burlaga, L. F., F. B. McDonald, N. F. Ness, and A. J. Lazarus (1991), Cosmic ray modulation: Voyager 2 observations, 1987-1988, J. Geophys. Res., 96, 3789-3799, doi:10.1029/90JA02245.
Cummings, A. C., E. C. Stone, F. B. McDonald, B. C. Heikkila, N. Lal, and W. R. Webber (2006), Termination shock particles spectral features, AIP Conf. Proc., 858, 86-91, doi:10.1063/1.2359310.

Giacalone, J., and J. R. Jokipii (2006), Energetic particle intensities and anisotropies of charged particles accelerated at the solar wind termination shock, Astrophys. J., 649, L137-L140, doi:10.1086/508463.

Gurnett, D. A., W. S. Kurth, S. C. Allendorf, and R. L. Poynter (1993), Radio emission from the heliopause triggered by an interplanetary shock, Science, 262, 199-203, doi:10.1126/science.262.5131.199.

Intriligator, D. S., W. Sun, M. Dryer, C. D. Fry, C. Deehr, and J. Intriligator (2005), From the Sun to the outer heliosphere: Modeling and analyses of the interplanetary propagation of the October/November (Halloween) 2003 solar events, J. Geophys. Res., 110, A09S10, doi:10.1029/2004JA010939.

Intriligator, D. C., W. Sun, A. Rees, T. Horbury, W. R. Webber, C. Deehr, T. Detman, M. Dryer, and J. Intriligator (2008), Three-dimensional simulations of shock propagation in the heliosphere and beyond, AIP Conf. Proc., 1039, 375-383, doi:10.1063/1.2982474.

Lookwood, J. A. (1960), An investigation of the Forbush decreases in the cosmic radiation, J. Geophys. Res., 65, 3859-3865, doi:10.1029/ JZ065i012p03859.

McDonald, F. B., W. R. Webber, E. C. Stone, A. C. Cummings, B. C. Heikkila, and N. Lal (2006), Voyager observations of galactic and anomalous cosmic rays in the heliosheath, AIP Conf. Proc., 858, 79-85, doi:10.1063/1.2359309.

Müller, H. R., G. P. Zank, P. Faurot-Pigion, V. Jivariyarej, R. Mietiso, and F. Shafi (2006), Effects of variable interstellar environments of the heliosphere, AIP Conf. Proc., 858, 33-38, doi:10.1063/1.2359302.

Richardson, J. D., C. Wang, J. C. Kasper, and Y. Liu (2005), Propagation of the October/November 2003 CMEs through the heliosphere, Geophys. Res. Lett., 32, L03S03, doi:10.1029/2004GL020679.

Richardson, J. D., Y. Liu, C. Wang, D. J. McComas, E. C. Stone, A. C. Cummings, L. F. Burlaga, M. H. Acuna, and N. F. Ness (2006), Source and consequences of a large shock near 79 AU, Geophys. Res. Lett., 33, L23107, doi:10.1029/2006GL027983.

Richardson, J. D., J. A. Kasper, C. Wang, J. W. Belcher, and A. J. Lazarus (2008), Cool heliosheath plasma and deceleration of the upstream solar wind at the termination shock, Nature, 454, 63-66, doi:10.1038/ nature 07024 .

Stone, E. C., A. C. Cummings, F. B. McDonald, B. C. Heikkila, N. Lal, and W. R. Webber (2008), An asymmetric solar wind termination shock, Nature, 454, 71-74, doi:10.1038/nature07022.

Washimi, H., G. P. Zank, A. Hu, T. Tanakan, and K. Mankato (2007), A forecast of the heliospheric termination shock position by threedimensional MHD simulations, Astrophys. J., 670, L139-L142, doi:10.1086/524358.

Webber, W. R. (2005), An empirical estimate of the heliospheric termination shock location with application to the intensity increase of $\mathrm{MeV}$ protons seen at Voyager 1 in 2002-2003, J. Geophys. Res., 110, A10103, doi:10.1029/2005JA011209

Webber, W. R., A. C. Cummings, F. B. McDonald, E. C. Stone, B. Heikkila, and N. Lal (2007), Passage of a large interplanetary shock from the inner heliosphere to the heliospheric termination shock and beyond: Its effects on cosmic rays at Voyagers 1 and 2, Geophys. Res. Lett., 34, L20107, doi:10.1029/2007GL031339.

A. C. Cummings, Downs Laboratory, California Institute of Technology, Mail Code 290-17, Pasadena, CA 91125, USA.

B. Heikkila, NASA Goddard Space Flight Center, GSFC Building 26/123, Greenbelt, MD 20771, USA.

N. Lal, NASA Goddard Space Flight Center, Code 644, Room S203, Building 2, Greenbelt, MD 20771, USA.

F. B. McDonald, Institute of Physical Science and Technology, University of Maryland, Computer Space Science Building 3245, College Park, MD 20742, USA.

E. C. Stone, Downs Laboratory, California Institute of Technology, MS 220-47, Pasadena, CA 91125, USA.

W. R. Webber, Department of Astronomy, New Mexico State University, P.O. Box 3001, 1320 Frenger Street, Las Cruces, NM 88003, USA. (bwebber@nmsu.edu) 